\title{
A IMPORT NCIA DO DESENVOLVIMENTO DE SOFT SKILLS NA FORMAÇÃO E INSERÇÃO DO PROFISSIONAL DA ENGENHARIA NO MERCADO DE TRABALHO
}

DOI: 10.37702/2175-957X.COBENGE.2021.3641

\author{
AMANDA DA SILVA SANTOS - AMANDASSAMANDA70@GMAIL.COM \\ UNIVERSIDADE FEDERAL DE ALAGOAS \\ RUA CAPITÃO CORREIA 80 \\ 57044-050 - MACEIÓ - AL
}

Andressa Tairine Evangelista - andressatairine@hotmail.com

Senai Cimatec

Rua Camuripeba; Cond. São Francisco 12

41650-035 - Salvador - BA

Maria Santos Coelho - maria.coelho93@gmail.com

Instituto Federal da Bahia

Rua Boulevard Seara 07

40301-010 - Salvador - BA

Ana Luisa Almeida Santos de Oliveira - ana.alavanca@gmail.com

Universidade Federal da Bahia

Avenida Paulo VI 111

41810-000 - Salvador - BA

Resumo: Na formação em engenharia, o conhecimento técnico faz-se presente em toda a graduação, com foco na resolução de problemas. No entanto, o profissional de engenharia possui um perfil abrangente e multidisciplinar, o qual o permite ocupar diversos cargos que exigem muito mais do que o conhecimento técnico-científico. Estudos apontam que em vagas de trabalho são pedidas cada vez mais como pré-requisitos as soft skills para ocupar diferentes cargos em distintos níveis hierárquicos. O referido trabalho traz uma abordagem da importância do desenvolvimento de habilidades interpessoais na formação do profissional da engenharia, por meio de uma visão ampla (observando as necessidades gerais do mercado) e de uma específica (direcionada aos profissionais da engenharia), a fim de trazer uma análise mais abrangente sobre 


\section{(C. COBENGE

as reais necessidades das soft skills para aumento e permanência da empregabilidade. Também evidencia a importância de startups de educação como forma de suprir a necessidade dos alunos de engenharia e preparação para ingressar no mercado de trabalho.

Palavras-chave: Soft skills, Mercado de trabalho, Engenharia, Educação, Explorer. 


\section{A IMPORTÂNCIA DO DESENVOLVIMENTO DE SOFT SKILLS NA FORMAÇÃO E INSERÇÃO DO PROFISSIONAL DA ENGENHARIA NO MERCADO DE TRABALHO}

\section{INTRODUÇÃO}

A engenharia se transforma de acordo com a evolução da tecnologia e as necessidades da sociedade, assim como o mercado de trabalho que também está em constante construção e adaptação. Pensamento lógico, agilidade na resolução de problemas, conhecimento na operação de máquinas e ferramentas são apenas algumas das hard skills (habilidades técnicas) que se espera de um profissional da engenharia, independente de qual área em específico ele tem expertise. No entanto, apenas essas habilidades técnicas não têm se mostrado suficientes para classificar um profissional como "bom" engenheiro (LIMA \& PORTO, 2018).

As soft skills são as habilidades relacionadas ao comportamento humano, seja individual, seja em sociedade (DEAN \& EAST, 2019). Inteligência emocional e autoconhecimento são pontos-chave para desenvolver requisitos que são cobrados nas diversas vagas de trabalho oferecidas por grandes empresas, a nível nacional e internacional (CIMATTI, 2016). O desenvolvimento do perfil de liderança, saber fazer uma tomada de decisão e gerenciar pessoas são tarefas que podem custar energia e tempo quando o encarregado por essas responsabilidades não consegue lidar com conflitos interpessoais e tem falhas de comunicação, por exemplo.

Estabelecer comunicação clara e objetiva é de grande importância para que o fluxo de informações ocorra da forma mais fluida e coerente possível. Faz-se necessário o entendimento de todo o processo e de todas as pessoas que nele atuam, a fim de compreender a importância de uma boa interação para a melhor execução das atividades determinadas. Estar atento ao impacto das suas ações no trabalho do outro colaborador e no sistema como um todo é indispensável para um melhor funcionamento da empresa e gerar os resultados esperados (PEDROSA, 2020). Na Figura 1, pode-se observar alguns exemplos de soft e hard skills.

Figura 1 - Soft e hard skills

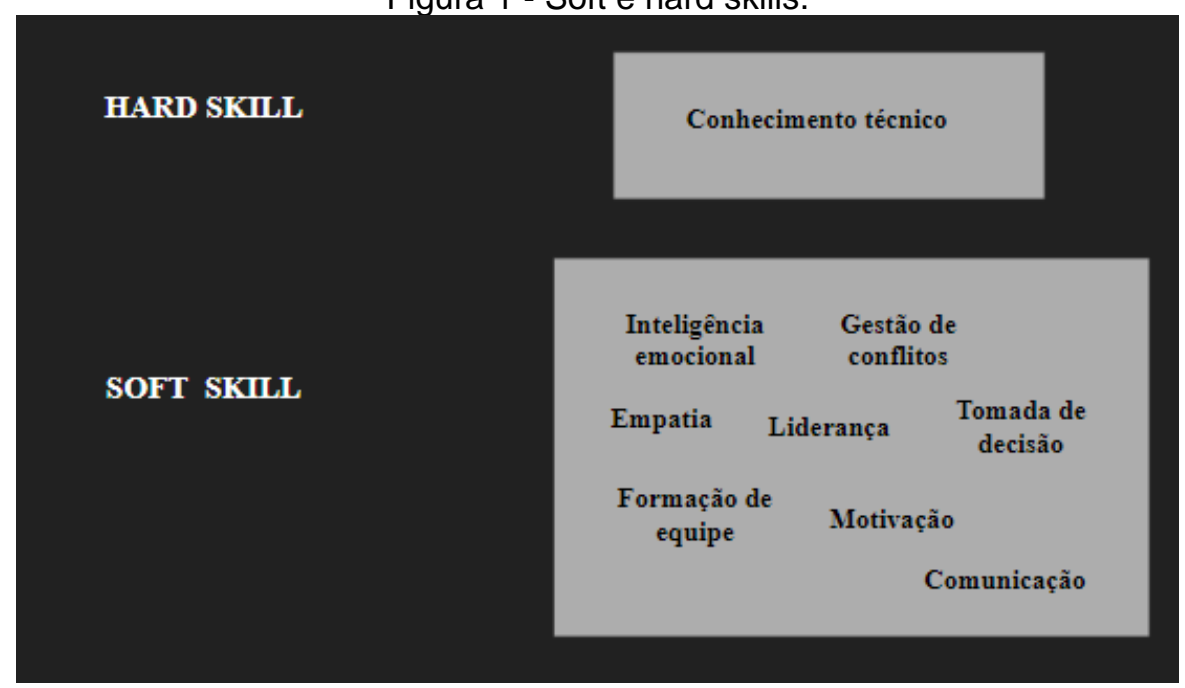

Fonte: Pedrosa, 2020. 
É preciso ser soft com as pessoas, sabendo lidar e mediar possíveis conflitos, e hard com os problemas, resolvendo-os de forma efetiva (PEDROSA, 2020). As vagas de trabalho estão exigindo muito além de cursos de capacitação, domínio de metodologias específicas ou apenas portar um diploma. É necessário saber gerir situações e se relacionar bem com as pessoas no ambiente laboral e, para isso, o autoconhecimento surge como um ponto de partida muito importante para alavancar essas habilidades (CIMATTI, 2016).

Assim, o referido trabalho tem como objetivo principal avaliar a importância do desenvolvimento de soft skills durante a formação nos cursos de engenharia. A pesquisa foi baseada em artigos, teses e dissertações presentes na literatura para possibilitar uma análise da necessidade do aperfeiçoamento de habilidades interpessoais. Desta forma, como objetivos específicos deste trabalho, têm-se:

- Analisar as exigências do mercado de trabalho atual requeridas ao profissional de engenharia;

- Apresentar o Explorer, programa de aceleração de carreira criado pela startup de educação O Que Aprendi na Engenharia (OQAE), com o propósito de suprir as habilidades exigidas dos alunos e recém-formados de engenharia e prepará-los para os reais desafios do mercado de trabalho.

\section{DESENVOLVIMENTO}

Alguns aspectos relevantes foram escolhidos para discutir sobre o tema, a fim de proporcionar uma análise mais ampla sobre o assunto abordado.

\subsection{Grade curricular dos cursos de engenharia}

Segundo o site Educamundo (2020), são listadas como as 10 primeiras faculdades mais renomadas de engenharia no Brasil as expostas na Tabela 1, assim como as suas respectivas notas associadas à classificação do Instituto Nacional de Estudos e Pesquisas Educacionais Anísio Teixeira (INEP), órgão do Ministério da Educação e Cultura (MEC). A posição que a universidade ocupa no ranking (classificação) depende da nota que ela obtém, após serem feitas avaliações realizadas pelo INEP, e varia de 0 (menor nota) até 5 (maior nota).

Tabela 1 - As 10 universidades de engenharia com maior nota do MEC no Brasil.

\begin{tabular}{c|c|c|c}
\hline Faculdade & Curso & Cidade/Estado & $\begin{array}{c}\text { Nota } \\
\text { (CPC) }\end{array}$ \\
\hline $\begin{array}{c}\text { Universidade Estadual de } \\
\text { Campinas (Unicamp) }\end{array}$ & Engenharia (geral) & Campinas/SP & 4,8539 \\
\hline $\begin{array}{c}\text { Instituto Militar de Engenharia } \\
\text { (IME) }\end{array}$ & Engenharia (geral) & Rio de Janeiro/RJ & 4,7056 \\
\hline $\begin{array}{c}\text { Instituto Tecnológico de } \\
\text { Aeronáutica (ITA) }\end{array}$ & Engenharia (geral) & $\begin{array}{c}\text { São José dos } \\
\text { Campos/SP }\end{array}$ & 4,6809 \\
\hline $\begin{array}{c}\text { Universidade do Vale do Rio } \\
\text { dos Sinos (Unisinos) }\end{array}$ & $\begin{array}{c}\text { Engenharia de } \\
\text { Alimentos }\end{array}$ & São Leopoldo/RS & 4,6276 \\
\hline $\begin{array}{c}\text { Pontifícia Universidade } \\
\text { Católica do Rio de Janeiro } \\
\text { (PUC-RJ) }\end{array}$ & Engenharia (geral) & Rio de Janeiro/RJ & 4,6189 \\
\hline
\end{tabular}




\begin{tabular}{c|c|c|c}
\hline $\begin{array}{c}\text { Universidade Federal de São } \\
\text { Paulo (Unifesp) }\end{array}$ & $\begin{array}{c}\text { Engenharia da } \\
\text { Computação }\end{array}$ & $\begin{array}{c}\text { São José dos } \\
\text { Campos/SP }\end{array}$ & 4,5826 \\
\hline $\begin{array}{c}\text { Universidade Salvador } \\
\text { (Unifacs) }\end{array}$ & $\begin{array}{c}\text { Engenharia de } \\
\text { Controle e } \\
\text { Automação }\end{array}$ & Salvador/BA & 4,4823 \\
\hline $\begin{array}{c}\text { Universidade Federal do } \\
\text { Maranhão (UFMA) }\end{array}$ & $\begin{array}{c}\text { Engenharia da } \\
\text { Computação }\end{array}$ & São Luís/MA & 4,4593 \\
\hline $\begin{array}{c}\text { Universidade do Vale do Rio } \\
\text { dos Sinos (Unisinos) }\end{array}$ & $\begin{array}{c}\text { Engenharia de } \\
\text { Controle e } \\
\text { Automação }\end{array}$ & São Leopoldo/RS & 4,4478 \\
\hline $\begin{array}{c}\text { Estadual Paulista Júlio de } \\
\text { Mesquita Filho (Unesp) }\end{array}$ & Engenharia Ambiental & $\begin{array}{c}\text { São José dos } \\
\text { Campos/SP }\end{array}$ & 4,4208 \\
\hline Fonte: Educamundo, 2020 & \multicolumn{3}{|c}{} \\
\hline
\end{tabular}

Quadro 1 - Grade curricular geral dos cursos de engenharia das 10 universidades com maior nota do MEC no Brasil.

\begin{tabular}{|c|c|}
\hline FACULDADE & GRADE CURRICULAR OBRIGATÓRIA \\
\hline $\begin{array}{l}\text { Universidade Estadual de Campinas } \\
\text { (Unicamp) }\end{array}$ & \multirow{10}{*}{$\begin{array}{l}\text { Química (a depender do curso) } \\
\text { Estágio supervisionado } \\
\text { Matérias técnicas específicas da área } \\
\text { de formação } \\
\text { Trabalho de conclusão de curso }\end{array}$} \\
\hline Instituto Militar de Engenharia (IME) & \\
\hline Instituto Tecnológico de Aeronáutica (ITA) & \\
\hline $\begin{array}{l}\text { Universidade do Vale do Rio dos Sinos } \\
\text { (Unisinos) }\end{array}$ & \\
\hline $\begin{array}{c}\text { Pontifícia Universidade Católica do Rio de } \\
\text { Janeiro (PUC-RJ) }\end{array}$ & \\
\hline Universidade Federal de São Paulo (Unifesp) & \\
\hline Universidade Salvador (Unifacs) & \\
\hline Universidade Federal do Maranhão (UFMA) & \\
\hline $\begin{array}{l}\text { Universidade do Vale do Rio dos Sinos } \\
\text { (Unisinos) }\end{array}$ & \\
\hline $\begin{array}{l}\text { Estadual Paulista Júlio de Mesquita Filho } \\
\text { (Unesp) }\end{array}$ & \\
\hline
\end{tabular}

Fonte: Autores, 2021.

A ementa dos cursos de engenharia tem como padrão matérias do ciclo básico e do ciclo profissional, sendo o estágio a primeira oportunidade de vivência prática, onde são aplicados os conhecimentos técnicos aprendidos na graduação. Esse período de estágio também é o momento de aprendizado de habilidades 
interpessoais, o qual o estudante terá de lidar com desafios reais. O que se percebe em comum entre as grades curriculares dos cursos citados na Tabela 1 e no Quadro 1, é a ausência de disciplinas voltadas ao treinamento de habilidades soft, o que gera a necessidade de buscar capacitação fora do ambiente acadêmico.

\subsection{Habilidades exigidas pelo mercado de trabalho}

Algumas habilidades como comunicação efetiva, pensamento criativo, resiliência, empatia, liderança e ética no trabalho são listadas como as mais requisitadas pelo mercado de trabalho, independente do cargo que se deseja ocupar (SPANGA, 2017). Segundo Lima \& Porto (2018), alguns dos aspectos mais cobrados em processos seletivos para engenheiros de software, por exemplo, são evidenciados no Quadro 2.

Quadro 2 - Aspectos cobrados em processos de seleção para profissionais da engenharia de software.

\begin{tabular}{|c|c|}
\hline FATOR & JUSTIFICATIVA \\
\hline Atitude & $\begin{array}{c}\text { A equipe do projeto deve ter uma atitude positiva a respeito do } \\
\text { seu trabalho e ter disposição em aprender novas técnicas. Esse } \\
\text { é um fator muito importante, mas, muitas vezes, difícil de avaliar } \\
\text { em uma entrevista. }\end{array}$ \\
\hline $\begin{array}{l}\text { Capacidade de } \\
\text { comunicação }\end{array}$ & $\begin{array}{c}\text { Necessidade dos membros da equipe de se comunicarem } \\
\text { verbalmente e por escrito com outros engenheiros, gerentes e } \\
\text { clientes. }\end{array}$ \\
\hline $\begin{array}{l}\text { Experiência na } \\
\text { linguagem de } \\
\text { programação }\end{array}$ & $\begin{array}{l}\text { Normalmente, é importante para projetos de curta duração, } \\
\text { quando há pouco tempo para aprender uma nova linguagem. }\end{array}$ \\
\hline $\begin{array}{l}\text { Experiência na } \\
\text { plataforma }\end{array}$ & $\begin{array}{c}\text { Aspecto importante se uma programação de baixo nível estiver } \\
\text { envolvida. Do contrário, esse aspecto não se apresenta como } \\
\text { muito importante. }\end{array}$ \\
\hline $\begin{array}{l}\text { Experiência no } \\
\text { domínio da } \\
\text { aplicação }\end{array}$ & $\begin{array}{l}\text { Para o desenvolvimento bem-sucedido de um projeto, os } \\
\text { desenvolvedores têm que compreender bem o domínio da } \\
\text { aplicação. }\end{array}$ \\
\hline $\begin{array}{l}\text { Formação } \\
\text { educacional }\end{array}$ & $\begin{array}{l}\text { Fornece um indicador dos conhecimentos básicos que o } \\
\text { candidato deve saber e da sua capacidade de aprender. Esse } \\
\text { fator se torna cada vez mais irrelevante à medida que os } \\
\text { engenheiros ganham experiência em diversos projetos. }\end{array}$ \\
\hline Personalidade & $\begin{array}{l}\text { Importante atributo, mas de difícil avaliação. Nenhum tipo } \\
\text { particular de personalidade é mais ou menos adequado à } \\
\text { engenharia de software. }\end{array}$ \\
\hline
\end{tabular}

Fonte: Lima \& Porto, 2018 (adaptado).

A partir da observação do Quadro 2, é possível notar que a competência técnica como a experiência no domínio da aplicação, por exemplo, é importante para 
que o projeto executado seja bem-sucedido. Porém, as outras hard skills como experiência na plataforma e experiência na linguagem de programação apresentamse como condicionais, visto que serão exigidas a depender do tipo de atividade e projeto a ser desenvolvido. Em contrapartida, quando observada a justificativa para os fatores capacidade de comunicação, atitude e personalidade, pode-se notar que eles são essenciais para que haja um bom relacionamento no ambiente de trabalho, comunicação clara e fluida com os colaboradores da empresa, assim como com os clientes.

Em uma visão mais ampla de formação profissional (independente da área), um estudo voltado para o treinamento de soft skills com alunos do curso de administração mostrou que, quando oferecido o treinamento de habilidades interpessoais, foi possível melhorar significativamente as competências sociais dos alunos envolvidos na análise (JOHN, 2009). Neste trabalho, foram avaliados componentes de habilidades pessoais, grupo experimental, grupo de controle, tempo e nível de significância. É possível observar a relação entre a média dos valores que foram mensurados no experimento em relação aos grupos, como mostrado na Figura 2.

Figura 2 - Relação entre a média de pontos mensurados no experimento em relação ao grupo de controle e grupo experimental para cada soft skill analisada.

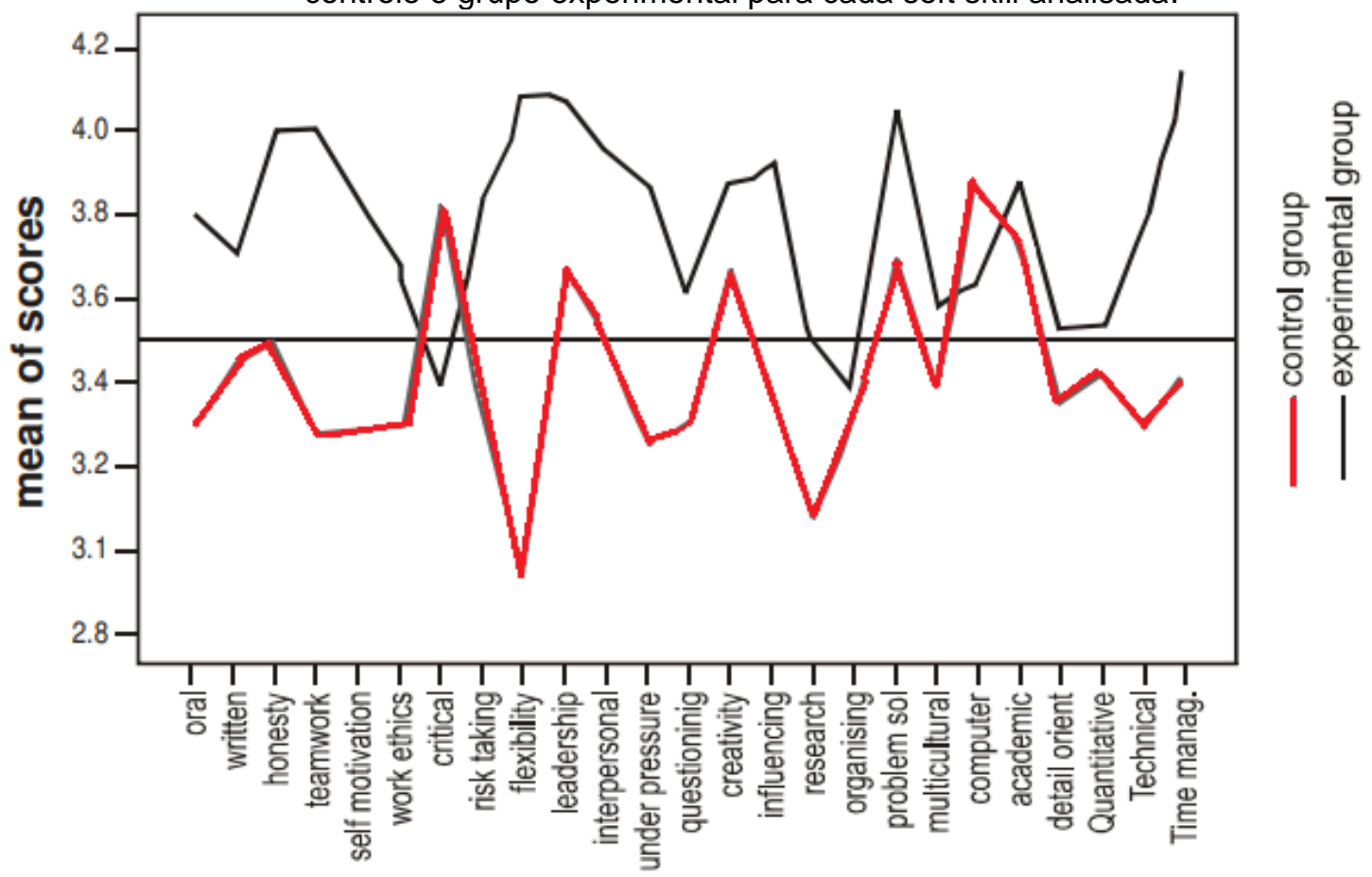

Fonte: John, 2009.

Este estudo apontou que, ao desenvolver essas soft skills por meio de um programa de treinamento, foi possível observar melhorias significativas das habilidades sociais desses alunos, de modo geral. Partindo disso, é possível aumentar a empregabilidade desses alunos em cargos de alta gestão ou de liderança e gerenciamento de equipes, visto que eles estarão mais preparados para lidar com diferentes situações, além de saber se relacionar melhor no ambiente laboral. 


\subsection{Habilidades do futuro}

Segundo o World Economic Forum (2016), algumas habilidades foram listadas como as que serão mais cobradas no mercado de trabalho até 2030 e, dentre elas, estão listadas: pensamento crítico e aprendizagem ativa, criatividade e originalidade, resolução de problemas complexos, flexibilidade cognitiva, inteligência emocional, trabalho em equipe, gestão de pessoas, negociação, tomada de decisões e orientação a serviços. Assim, em uma visão ampla e a nível mundial, é possível observar que o desenvolvimento de habilidades soft se faz presente como requisito para vagas de emprego, de diferentes áreas, incluindo a engenharia.

\subsection{Capacitação direcionada para engenheiros}

O Que Aprendi na Engenharia (OQAE) é uma Startup EdTech dedicada a promover Experiências que preparam engenheiros(as) e futuros(as) engenheiros(as) para os reais desafios do Mercado de Trabalho. Diante da necessidade de suprir as habilidades requeridas no meio profissional e que não são ensinadas em sala de aula durante a graduação, O OQAE criou o Explorer: programa (experiência) de aceleração de carreira para universitários(as) e recém-formados(as) de engenharia. Atualmente, o Explorer é dividido em três Trilhas (OQAE, 2021) :

- Soft skills: onde doze competências comportamentais, listadas como habilidades do futuro, são ensinadas por meio de aulas e exercícios práticos, baseando-se, primeiramente, no talento de cada pessoa que acessa ao programa;

- Hard skills: nessa Trilha são ministrados treinamentos de habilidades técnicas exigidas na engenharia, por profissionais que as vivenciam na prática;

- Carreiras: uma biblioteca de experiências práticas. São cases (entrevistas) com diversos especialistas da engenharia, que atuam em diferentes áreas e posições no mercado de trabalho.

Além das Trilhas supracitadas, o Explorer proporciona mentorias coletivas com os seus membros e oferece grupos focais para diferentes objetivos de carreira da engenharia: a) quem está buscando aprovação em estágio ou trainee; b) quem está em busca de efetivação/promoção no cargo; c) quem está em transição de carreira; e d) para quem deseja empreender.

Dessa forma, o Explorer consegue capacitar o engenheiro não apenas nas hards skills que são exigidas ao profissional, mas também nas soft skills, que são cada vez mais requisitadas no mercado de trabalho. Assim, o desenvolvimento e aprimoração dessas habilidades comportamentais por meio desta experiência, fazse extremamente importante, uma vez que configuram um gap (lacuna) nas grades curriculares das universidades de engenharia (Quadro 1) e são requeridas no cotidiano do profissional em qualquer empresa/organização.

\section{CONSIDERAÇÕES FINAIS}

Diante do exposto, é possível observar que as matérias técnico-científicas são de grande importância para caracterizar o perfil do profissional da engenharia e permitir que, com essa habilitação, ele seja capaz de resolver problemas e desenvolver atividades referentes à sua área de atuação. Porém, apesar dessa base importante, os estudos apontam que as soft skills apresentam-se como essenciais para criar e manter boas relações sociais, gerenciar equipes e para promover o 
autoconhecimento. Ademais, o desenvolvimento de habilidades interpessoais oferecidas por meio do Explorer proporciona o aumento das chances de empregabilidade, visto que as pessoas estarão mais preparadas para lidar com as mudanças as quais o mercado de trabalho sofre constantemente. Dessa forma, é evidenciado, assim, a importância dessa educação complementar oferecida por Startups de educação como solução para suprir essa lacuna.

\section{REFERÊNCIAS}

CIMATTI, B. Definition, development, assessment of soft skills and their role for the quality of organizations and enterprises. International Journal for Quality Research, v. 10, n.1, p. 97-130, 2016.

DEAN, S. A.; EAST, J. I. Soft Skills Needed for the 21st-Century Workforce.International Journal of Applied Management and Technology, v. 18, n. 1, p. 17-32, 2019.

Educamundo. Os 50 melhores cursos de engenharia do Brasil, segundo o MEC. Disponível em: https://www.educamundo.com.br/blog/melhores-cursosengenharia-brasil. Acesso em 21 de abr. de 2021.

JOHN, J. Study on the Nature of Impact of Soft Skills Training Programme on the Soft Skills Development of Management Students. Pacific Business Review, p. 19-27, 2009.

LIMA, Tiago; PORTO, Josiane . Análise de Soft Skills na Visão de Profissionais da Engenharia de Software. In: IV Workshop sobre aspectos sociais, humanos e econômicos de software (WASHES), 2019, Belém. Anais. Porto Alegre: Sociedade Brasileira de Computação. Disponível em: https://doi.org/10.5753/washes.2019.6407. Acesso em 20 abr. 2021.

O QUE APRENDI NA ENGENHARIA. Explore. Disponível em: https://oqueaprendinaengenharia.com/explorer/. Acesso em 08 de mai. de 2021.

PEDROSA, Valmir Albuquerque. Construindo pactos pelo uso da água. Edital 01/2020 Projeto 914BRZ2022. Out. de 2020.

SPANGA, J. D. 6 "Soft Skills" mais requisitadas pelo mercado. Disponível em: https://forbes.com.br/carreira/2017/07/6-soft-skills-mais-requisitadas-pelomercado/\#foto6. Acesso em 21 de abr. de 2021.

WORLD ECONOMIC FORUM. The Future of Jobs. Disponível em: https://www.weforum.org/reports/the-future-of-jobs. Acesso em 23 de abr. de 2021. 


\title{
THE IMPORTANCE OF SOFT SKILLS DEVELOPMENT IN THE TRAINING AND INSERTION OF THE ENGINEERING PROFESSIONAL IN THE LABOR MARKET
}

\begin{abstract}
In engineering training, technical knowledge is present throughout the undergraduate program, with a focus on problem solving. However, the engineering professional has a comprehensive and multidisciplinary profile, which allows him to occupy several positions that require much more than technical-scientific knowledge. Studies show that in job vacancies, soft skills are increasingly being asked as prerequisites to occupy different positions at different hierarchical levels. The referred work brings an approach of the importance of the development of interpersonal skills in the formation of the engineering professional, through a broad vision (observing the general needs of the market) and a specific one (directed to the engineering professionals), in order to bring a more comprehensive analysis of the real needs of soft skills to increase and maintain employability. It also highlights the importance of education startups as a way of meeting the needs of engineering students and preparing to enter the job market.
\end{abstract}

Key words: Soft skills, Labor market, Engineering, Education, Explorer. 